\title{
ANÁLISIS BIOMECÁNICO DE EL GESTO TÉCNICO EN BARRAS PARALEAS EN LA GIMNAS IA OLÍMPICA
}

\author{
BIOMECHANICAL ANALYSIS OF TECHNICAL GESTURE IN PARALLEL \\ BARS IN THE OLYMPIC GYMNAS TICS
}

\author{
Esp. Yesid Santafé Ramón, Ing. Luis Alberto Muñoz, MSc. Luis Enrique Mendoza. \\ Universidad de Pamplona, Grupo de Investigación en Ingeniería Biomédica GIBUP. \\ Ciudadela Universitaria, Pamplona, Norte de Santander. Tel (+775) 5685303, Ext. 144. \\ E-mail\{inglu ismb, luis.mendoza, yesid.santafe\}@unipamplona.edu.co
}

\begin{abstract}
Resumen: El presente artículo muestra los resultados iniciales del análisis biomecánico para estudio del gesto técnico en barras paralelas en deportistas de alto rendimiento en gimnasia olímpica. Se presentan medidas tales como: distancia, trayectoria y velocidad del movimiento. Además se muestra el seguimiento del movimiento y los análisis estáticos del movimiento usando el software Kinovea. Se demuestra que el software permite realizar análisis biomecánico en movimiento de barras paralelas en gimnasia olímpica.
\end{abstract}

Palabras clave: Biomecánica, Gesto Técnico, Cinemática.

\begin{abstract}
This paper presents the initial results of biomechanical analysis to study the technical gesture on parallel bars in high-performance athletes in Olympic gymnastics. Distance, trajectory and speed of movement: such measures as they arise. Besides tracking the movement and static motion analysis using the software Kinovea shown. It is shown that biomechanical analysis software allows moving parallel bars in gymnastics.
\end{abstract}

Keywords: Biomechanics, Technical Gesture, Kinematics.

\section{INTRODUCCIÓN}

La biomecánica no es un área reservada tan sólo para los entrenadores deportivos. Ésta es usada por diversas disciplinas que incluyen la biología, la fisiología, la medicina y la mecánica. Muchos profesionales -ingenieros, terapistas físicos, cirujanos ortopédicos o ingenieros aeroespaciales - hacen aplicaciones prácticas de la mis ma.

Hoy en día los ingenieros estudian las propiedades biológicas y materiales del cuerpo humano y sus aspectos electromecánicos para entendersu fenomenología. (Acero, 2009).

Para estudiar el movimiento humano, la biomecánica deportiva utiliza dos procedimientos: el análisis cuantitativo y el cualitativo (Acero, 2009).
El análisis cuantitativo implica la descripción de los movimientos del cuerpo o sus partes en términos numéricos. Tal cuantificación de las características del movimiento ayuda a eliminar las descripciones subjetivas ya que los datos son obtenidos mediante el uso de instrumentos.

El observador puede entonces usar esta cuantificación para explicar o describir la situación actual. Usualmente, este análisis tiene algunos inconvenientes como no ser económico por el uso de los instrumentos, requerir mucho tiempo o por la dificultad para llevar los instrumentos al campo

El análisis cualitativo intenta describir un movimiento en términos no numéricos. (Acero, 2009). 


\section{BARRAS PARALELAS}

El aparato consiste en dos barras de las mismas dimensiones, Fig1, que están paralelas y a la misma altura. Cada barra esta sujeta a dos bases verticales. Estas bases se ajustan a un estable marco metálico que permite la graduación para diferentes alturas. Las barras tienen $350 \mathrm{~cm}$ de largo y están separadas la una de la otra por un rango de $42 \mathrm{~cm}-52 \mathrm{~cm}$. La altura desde el piso es de $200 \mathrm{~cm}$ y desde el borde superior de la colchoneta es de $180 \mathrm{~cm}$. (Gymnastiko).

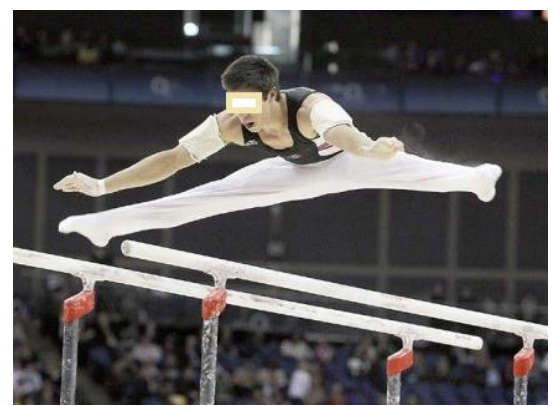

Fig. 1.Gesto Barras paralelas Fuente: site/gimnasiaolimicavaronil/aparatos

Las "barras paralelas", son un aparato utilizado sólo en la categoría masculina, y se contempla dentro de la modalidad de la gimnasia artística. En la fig 2 se describe un ejercicio de paralelas contemporáneo predominan los impulsos y elementos de vuelo seleccionados entre una gran variedad de grupos de elementos y realizados con transiciones continuas entre suspensiones y apoyos, de manera que refleje todo el potencial del aparato.( Gymnastiko)

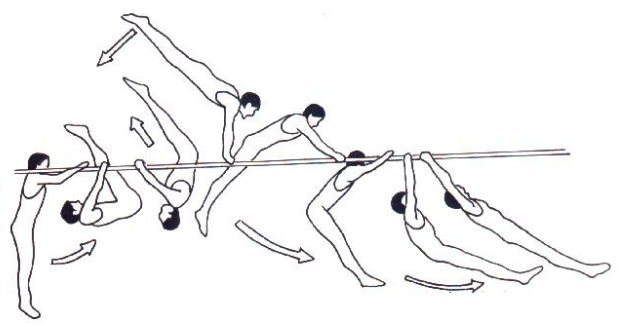

Fig.2.Movimiento Barras paralelas

Fuente: site/gimnasiaolimicavaronil/aparatos

Este aparato en general, requiere de un gran equilibrio y fuerza de brazos como de abdomen, ya que consta del constante impulso y posterior elevación de piernas para realizar las acrobacias o movimientos estipulados. (Aparatos).

El parámetro principal de la biomecánica necesario para el desarrollo y puesta en marcha del presente proyecto es la cinemática.

La cinemática: consiste en un conjunto de métodos que busca medir parámetros cinemáticos del movimiento, esto es, a partir de la adquisición de imágenes durante la ejecución del movimiento, se realiza el cálculo de las variables dependientes de los datos observados en las imágenes, como es el caso de la posición, orientación, velocidad y aceleración del cuerpo o de sus segmentos. (Mstdo. Stuart, Soares- 2012)

\section{GESTO TÉCNICO}

Todo movimiento corporal es necesario para manejar y controlar herramientas, máquinas, instrumentos, etc, a este tipo de movimiento se le conoce como gesto técnico (Tecnología 18, 2012). Las funciones principales de los gestos técnicos son:

- Conocer cómo se manejan las herramientas.

- Crear conciencia sobre cómo mejorar o transformar las herramientas y maquinas que se operen.

Los elementos considerados para su caracterización son:

- Movimiento Corporal: que se efectúa al realizar una acción sobre una maquina o herramienta.

- Potencia: es el grado de fuerza ejercida al realizar una acción para manejar una maquina o herramienta.

- Precisión: es la exactitud con la que se lleva a cabo un movimiento corporal o gesto técnico.

- Complejidad: es el grado de dificultad para realizar el gesto técnico o movimiento corporal.

- Estratégicas: son aquellas que involucran un análisis previo ante la toma de decisiones.

- Las Acciones de Control: son aquellas que permiten una conectividad y comunicación entre las acciones estratégicas y las instrumentales.

- Las Acciones Instrumentales: son aquellas que organizan los métodos adecuados para llevar a cabo una acción con el debido control.

\section{SOFTWARE DE ANÁLISIS}

Kinovea es un software de análisis de vídeo dedicado al deporte. Está dirigido principalmente a los entrenadores, atletas y profesionales médicos. También puede ser útil para ergonomía y en el estudio de animación, es de resaltar que dicho software se puede descargar de forma gratuita directamente desde la página web.(Kinovea) La fig 3 muestra un pantallazo de la interfaz de trabajo del software Kinovea. 
Revista Colombiana de

Tecnologías de Avanzada

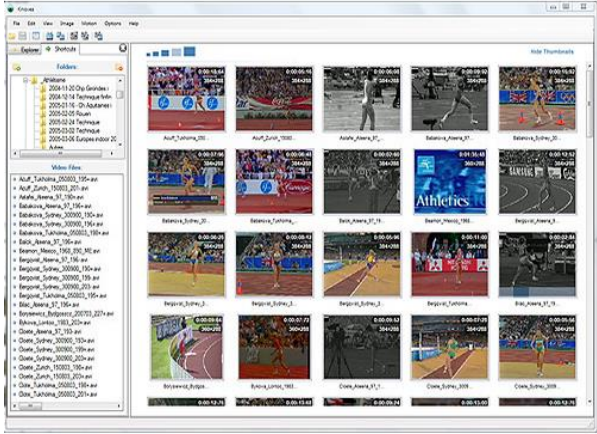

Fig.3.Interzas kinovea.

Fuente: kinovea.org/help/es/index.htm

El explorador de archivos integrado permite navegar por una colección de videos de forma visual. Los archivos compatibles se muestran como miniaturas animadas. Un gestor de acceso directo permite guardar los archivos favoritos de acceso frecuente para una búsqueda más rápida (Kinovea,). Los controles de vídeo permiten centrarse en una acción específica y estudiar la estructura del movimiento fotograma a fotograma o a cámara lenta. Kinovea reproduce casi cualquier archivo, por lo que no es necesario preocuparse por los formatos y codecs. (Kinovea,)

\section{ANÁLISIS}

El experimento y comprobación del funcionamiento de Kinovea se realizó besándose en una prueba con video general de gimnasia. Las fig 6, 7 y 8 representan las posibilidades de búsqueda y selección de archivos.

\subsection{Utilizando el software}

En este apartado se menciona como cargar archivos de video y descripción de algunos Botones

\subsubsection{Abrir Archivo}

\section{Opción1:}

Ir al menú Archivo luego dar click en abrir Archivo como se muestra en la figura.

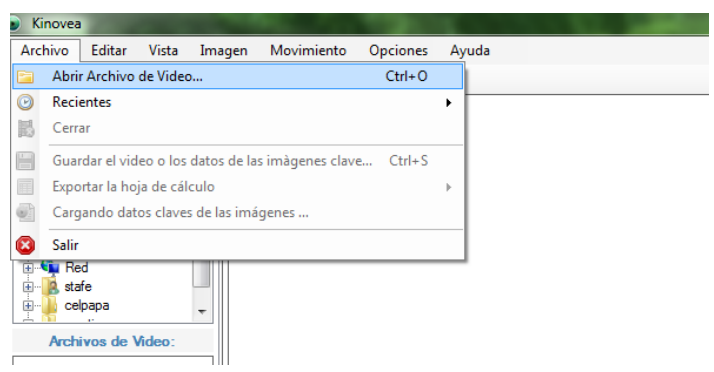

Fig. 4. Como abrir Archivo. Fuente: Autor
Posteriormente aparecerá otra ventana donde se debe buscar la carpeta que contiene nuestro video de interés

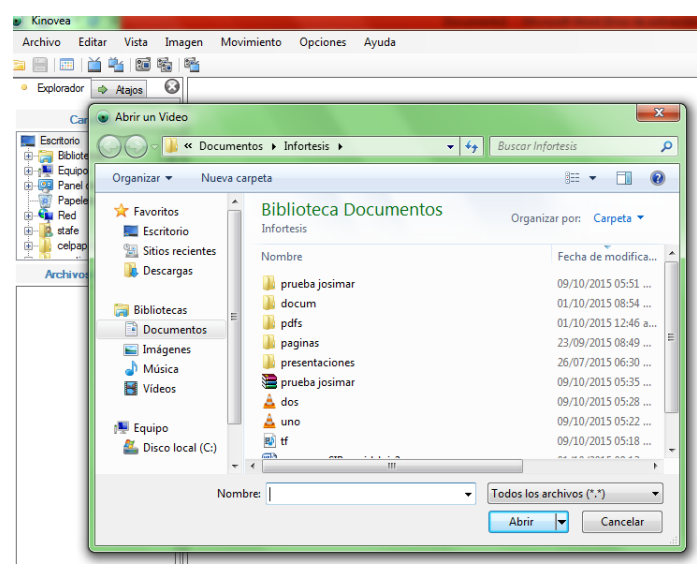

Fig. 5. Selecionar Archivo. Fuente: Autores.

\section{Opción 2:}

En la pestaña explorador se debe buscar la carpeta que contiene nuestro video de interés desplegando los símbolo $\mathrm{s}(+)$

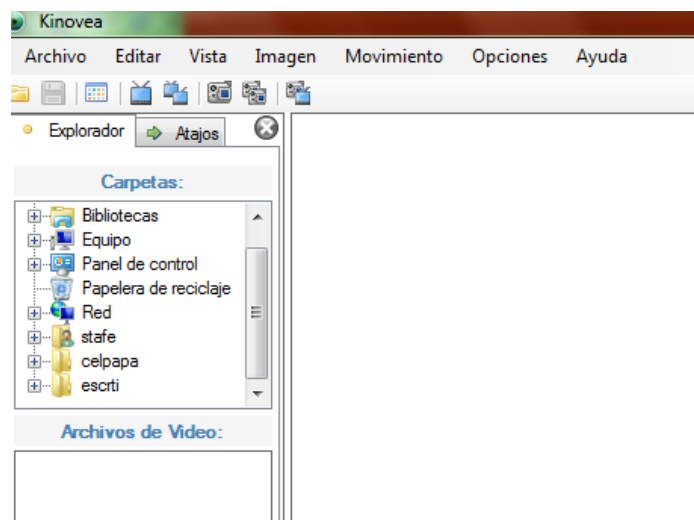

Fig.6. Seleccionar Archivo. Fuente: Autor.

A continuación aparecerá en la ventana archivos de video, los videos que tengamos en nuestra carpeta de interés fig.7.

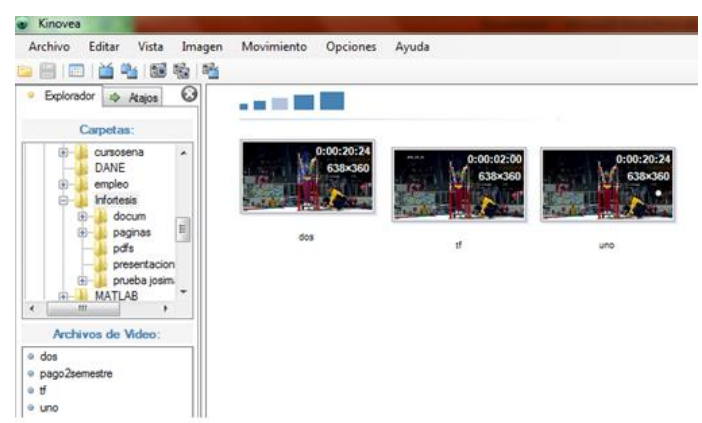

Fig.7. Seleccionarvideo. Fuente: Autor.

Para visualizarlos damos doble click en el nombre del archivo, o procedemos arrastrar el 
Revista Colombiana de

Tecnologías de Avanzada

archivo a la ventana que se encuentra a la derecha (en blanco), y aparecerá el entorno de reproducción con respectivos botones.

La siguiente información es tomada de la ayuda kinovea: Una vez que el video se abre, inicie la reproducción con los controles del reproductor fig. 8 o busque una ubicación arbitraria con el cursor de navegación.

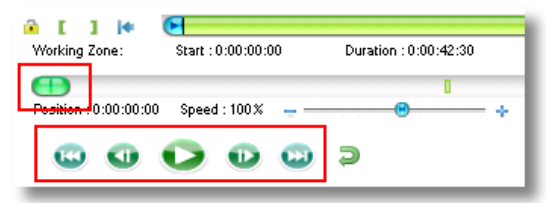

Fig.8. Botones Reproducción.

Fuente: kinovea.org/help/es/index.htm

\subsection{Especificar una zona de trabajo}

Sitúese en el punto que le interese y utilice el botón de inicio ubicado en la zona de trabajo. Inicie la reproducción y deje correr el vídeo has ta el final de la secuencia a analizar. Pulse el botón de fin ubicado en la zona de trabajo. Nota: El cursor de navegación es ahora más preciso, ya que se expande en la zona de trabajo. Si la reproducción está en modo de bucle, el video se repetirá dentro de la Zona de Trabajo.

\subsection{Aumentar el tamaño de la imagen}

Si la imagen parece demasiado pequeña, utilice los tiradores cuadrados pequeños en las esquinas de la imagen. Arrástrelos hasta que el tamaño de la imagen sea el deseado. También puede directamente hacer zoom en la imagen o utilizar la herramienta de lupa.

\subsection{Disminuir la velocidad la velocidad de reproducción}

Con el fin de mejorar el estudio del movimiento, reduzca la velocidad de reproducción utilizando el cursor de velocidad.

\subsection{Especificar una zona de trabajo para el análisis}

Con la ayuda de los cursores de selección $\Theta \mathrm{y} \boxplus$ establezca una pequeña zona de trabajo de todo el movimiento para realizar la observación. Una vez que la zona de trabajo es lo suficientemente pequeña, Kinovea cambiará almodo de análisis. En este modo los fotogramas que componen el vídeo se extraen fig. 9 a la memoria para un acceso más rápido.

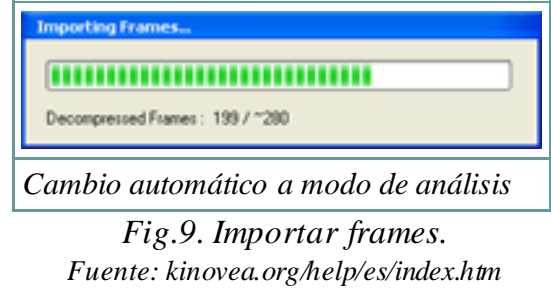

Las propiedades de la zona de trabajo correspondientes al modo de análisis se pueden configurar en el cuadro de diálogo preferencias, en la pestaña de la pantalla de Opciones. La duración predeterminada es de 12 segundos.

\subsection{Romper el movimiento}

El modo de análisis hace actualizar el vídeo en tiempo real cuando se utiliza el cursor de navegación. Esto da un control total sobre la línea de tiempo. También se puede utilizar la rueda del ratón para mover los fotogramas hacia delante o hacia atrás.

\subsection{Trabajo de seguimiento}

Los pasos a seguir en el seguimiento de un objeto (o una articulación del cuerpo) en Kinovea son los siguientes:

- Haga clic derecho en el objeto a seguir y utilice el menú seguir trayectoria.

- Mueva el vídeo hacia delante utilizando el botón de reproducción, el botón de Siguiente fotograma o la rueda del ratón.

- Ajuste la ubicación del punto cuando sea necesario durante la creación de la trayectoria.

- Para finalizar el seguimiento, haga clic derecho y utilice el menú terminar la edición de la trayectoria.
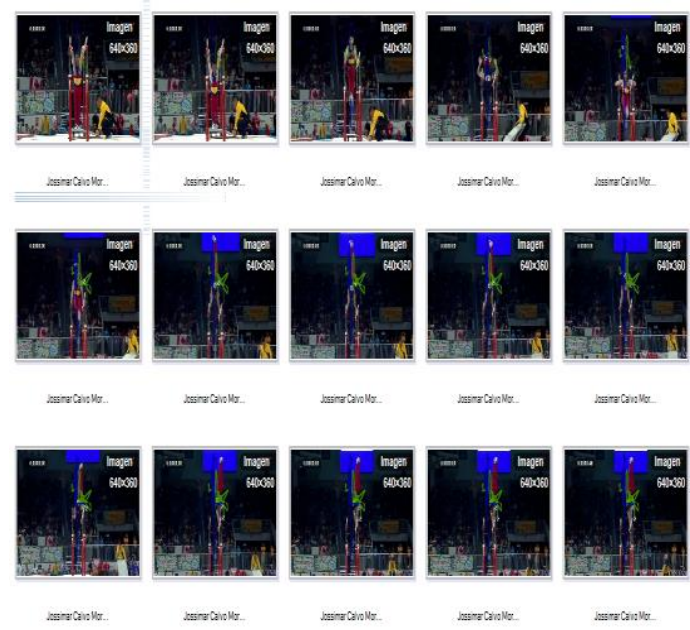

Fig.10. Seguimiento Trayectoria

Fuente: video sportv2 (Análisis Autor). 
El seguimiento es un proceso semi-automático. En la figura 10 la ubicación de los puntos se calcula de forma automática, pero puede ajustarlo en cualquier momento.

\subsection{Elegir el objeto a seguir}

Para mejorar los resultados del seguimiento automático, el objeto a seguir debe tener un buen contraste y una buena definición.

La forma del objeto no debe cambiar mucho durante el seguimiento. Puede considerar el uso de etiquetas de seguimiento o pegatinas reflectantes para mejorar los resultados

\subsection{Interactuar con el seguimiento}

Una vez que esté en la última imagen del punto sobre la que desea realizar el seguimiento de la trayectoria, finalice el seguimiento haciendo clic derecho en la herramienta y utilice el menú terminar la edición de la trayectoria.

Los rectángulos desaparecen. Todos los puntos quedan bloqueados. El seguimiento es ahora interactivo y puede saltar a cualquier imagen haciendo clic sobre el punto correspondiente. Fig.11.

Arrastre el objetivo a lo largo de la trayectoria para navegar por el movimiento.

Las imágenes que se muestran a continuación corresponden a seguimiento de trayectorias ubicando tres marcadores en muñecas mano Izquierda, mano derecha, y pie derecho con los siguientes colores de trayectorias:

- Mano Izquierda, color verde.

- Mano derecha, color amarillo.

- Pie derecho color azul.

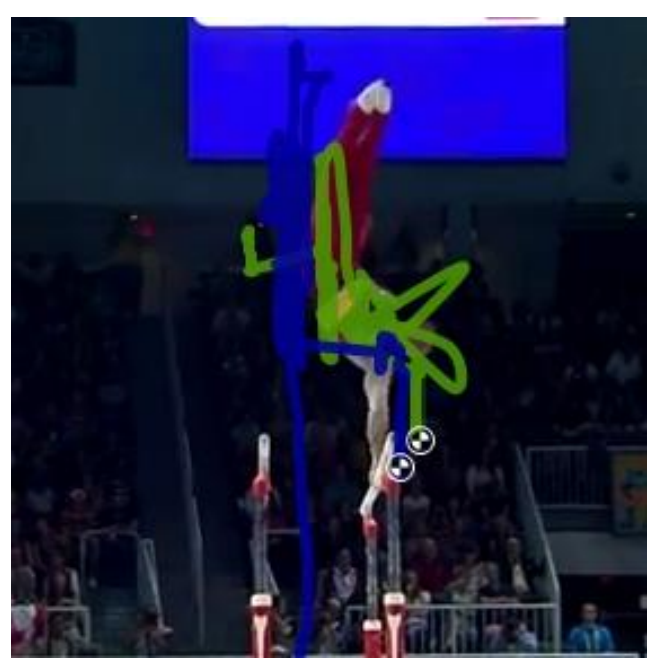

Fig.11. Seguimiento Trayectoria.

Fuente: video sportv2. (Análisis Autor).

\subsection{Configurar las opciones de visualización}

Haga clic derecho sobre la trayectoria y utilice el menú configuración para acceder a las opciones de visualización. Están disponibles las siguientes opciones: Modos de visualización.

La trayectoria puede estar en uno de estos tres modos:

- $\quad$ A ] - Trayectoria completa - La trayectoria se dibuja todo el tiempo. (opción por defecto).

- $\quad$ B] - Selección de la trayectoria alrededor de la imagen en curso- Sólo se dibuja una pequeña sección de la trayectoria, por lo general 25 imágenes en torno al punto actual.

- $\quad[C]$ - Etiqueta según la trayectoria - Sólo se dibuja una pequeña sección de la trayectoria, y la etiqueta definida se muestra en el punto actual.

\subsection{Medir}

Se puede visualizar información adicional sobre la distancia total o la velocidad eligiendo una de las opciones:

- Distancia - La distancia total entre el inicio de la trayectoria y el punto actual ver fig. 12.

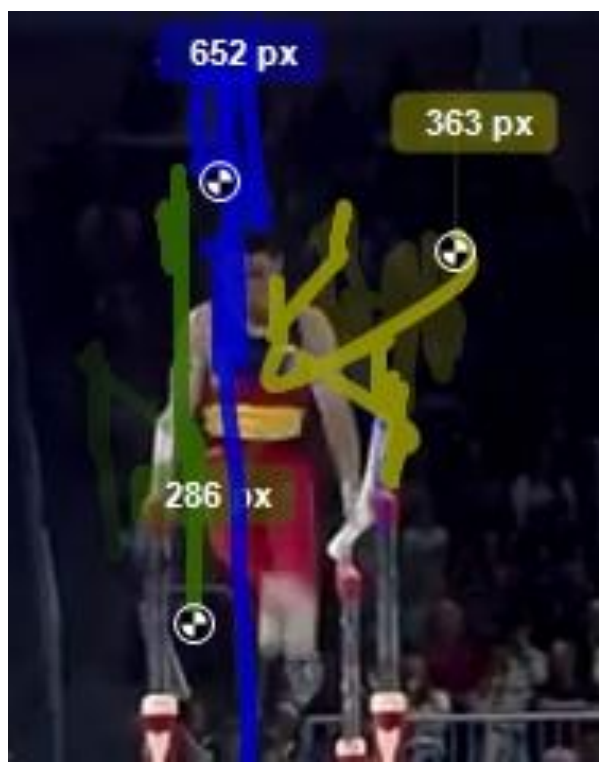

Fig.12. Seguimiento Distancias

Fuente: video sportv2. (Análisis Autor)

- Velocidad - La velocidad media del último segmento de la trayectoria fig. 13.

Para expresar la distancia y la velocidad en unidades reales, primero debe agregar una línea y establecer su longitud real, como se describe en medir distancias. 
Revista Colombiana de Tecnologías de Avanzada

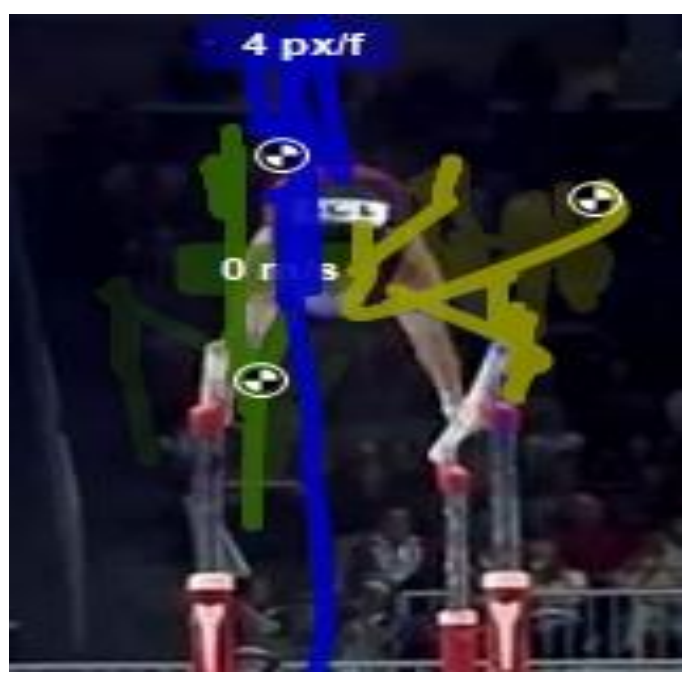

Fig. 13. Seguimiento Velocidades.

Fuente: video sportv2.(Análisis Autor).

Las coordenadas obtenidas se grafican a continuación Coords (x,y:px; t:time) fig. 14.

Donde el software toma 506 datos que equivalen a muestras de tiempo o duración del video de 20 segundos con 20 centésimas o 20200 milisegundos, en esta figura se muestra la relación coordenada " $\mathrm{x}$ " (colora azul) $\mathrm{y}$ coordenada " $y$ " (color verde). Se evidencia que los movimientos con mayor variación están en la coordenada " $y$ " mientras en " $x$ " hay poca variación y luego de aproximadamente la mitad de la rutina se estabiliza.

Trayectoria en un gráfico 3D con el tiempo como tercera dimensión.

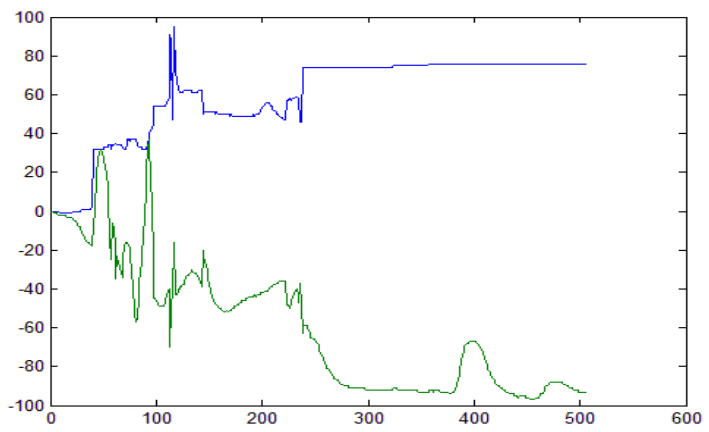

Fig. 14. Representación Coordenadas. Fuente: Autor

La figura 15 representa el uso de gnuplot para visualizar los datos de trayectoria a lo largo del eje del tiempo, los datos de trayectoria en un gráfico $3 \mathrm{D}$ con el tiempo como tercera dimensión.

La representación del tiempo debe ser numérica (por ejemplo: número de fotogramas o milisegundos totales).

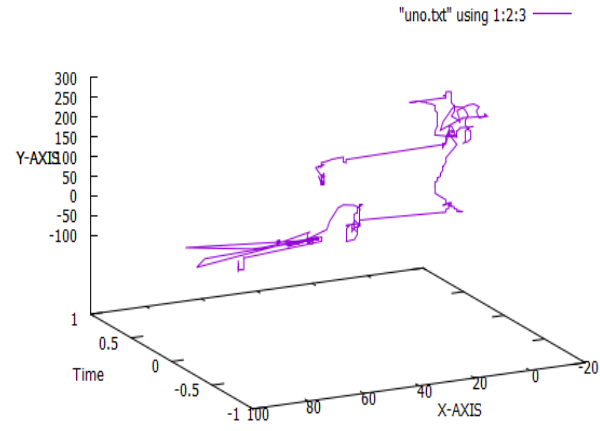

Fig. 15. Representación Trayectorias en Coordenadas.

Fuente: Autor.

\section{CONCLUS IONES}

La importancia de tener mediciones como distancia, velocidad y trayectoria permitirán en un futuro tener una herramienta que pueda medir el grado de perfección en el movimiento realizado. Por otro lado y haciendo uso de kinovea marca un trabajo posterior que se realizará como apoyo y validación para el correspondiente análisis de variables biomecánicas a estudiar.

La importancia del software utilizado tanto kinovea, como Open Source herramienta gráfica gnuplot son versiones libres, lo cual permite tanto buenas prestaciones como agilidad en los estudios.

\section{REFERENCIAS}

Acero J., Suárez R., Young-Hoo Kwon Gerald L. Scheirman, Hatfield D. (2009). "Biomecanica Deportiva y control del Entrenamiento", Universidad de Antioquia, Instituto Universitario de Educación Física, Funámbulo Editores, Medellín, Colombia.

Mstdo. Werlayne Stuart Soares Leite. (2012). "Biomecánica aplicada al deporte: contribuciones, perspectivas y desafíos"”. Revista Digital. Buenos Aires - Año 17 - No. 170.

Gutierres F, Canda A, Heras M. (2010). Análisis Valoración y monitorización del Entrenamiento de Alto Rendimiento Deperotivo, Editorial Enrique Lizalde, España

Miralles, F., García, M., Ramírez Mundilla, V., Navarro, E. "Análisis Biomecánico Del Doble Mortal Carpado De Salida En Paralelas De Jesús Carballo", Instituto Nacional de Educación Física de Madrid. Universidad Politécnica de Madrid. 


\section{SITIOS WEB}

Aparatos, Gimnasia Olimica Varonil, https://sites.google.com/site/gimnasiaolimica varonil/aparatos,

Tecnología 18. (2012) "Gesto Técnico", https://ms ms 06.wordpress.com/2012/11/14/gesto -tecnico/
Kinovea (2015), página official de software http://www.kinovea.org/

Gnuplot (2015), portable command-line driven graphing utility for Linux, OS/2, MS Windows, OSX, VMS, and many other platforms,http://www.gnuplot.info/

Gymnastikos (2013-2015), sitio dedicado a proveer información acerca del deporte de la gimnasia artística, http://gymnastikos .com/paralelas/ 